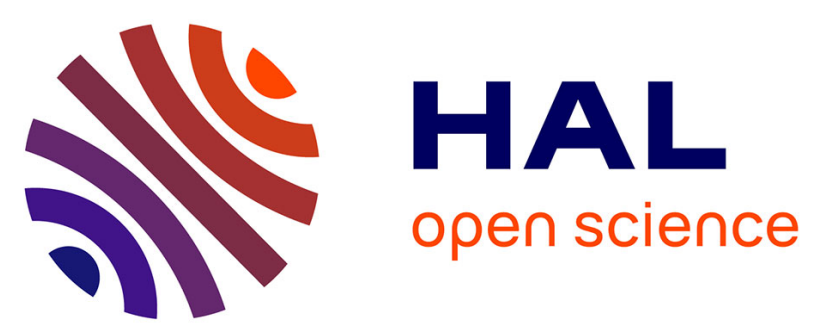

\title{
Weiss-Weinstein Bound and SNR Threshold Analysis for DOA Estimation with a COLD Array
}

Dinh Thang Vu, Alexandre Renaux, Remy Boyer, Sylvie Marcos

\section{To cite this version:}

Dinh Thang Vu, Alexandre Renaux, Remy Boyer, Sylvie Marcos. Weiss-Weinstein Bound and SNR Threshold Analysis for DOA Estimation with a COLD Array. IEEE International Workshop on Statistical Signal Processing - invited article, 2011, Nice, France. pp.13-16. hal-00577271

\section{HAL Id: hal-00577271 \\ https://hal.science/hal-00577271}

Submitted on 17 Mar 2011

HAL is a multi-disciplinary open access archive for the deposit and dissemination of scientific research documents, whether they are published or not. The documents may come from teaching and research institutions in France or abroad, or from public or private research centers.
L'archive ouverte pluridisciplinaire HAL, est destinée au dépôt et à la diffusion de documents scientifiques de niveau recherche, publiés ou non, émanant des établissements d'enseignement et de recherche français ou étrangers, des laboratoires publics ou privés.

\section{(1)(1) $\$(0)$}

Distributed under a Creative Commons Attribution - NonCommercial - ShareAlikel 4.0 


\title{
WEISS-WEINSTEIN BOUND AND SNR THRESHOLD ANALYSIS FOR DOA ESTIMATION WITH A COLD ARRAY
}

\author{
Dinh Thang VU, Alexandre RENAUX, Rémy BOYER, and Sylvie MARCOS \\ Laboratoire des Signaux et Systèmes (L2S), Université Paris-Sud XI, CNRS, SUPELEC \\ Gif-Sur-Yvette, France
}

\begin{abstract}
In the context of polarized sources localization using a cocentered orthogonal loop and dipole array, direction-of-arrival estimation performance in terms of mean square error are investigated. In order to evaluate these performance for both asymptotic and non-asymptotic scenarios (low number of snapshot and/or low signal to noise ratio) we derive closed-form expressions of the Weiss-Weinstein bound. The analysis is performed under both conditional and unconditional source signal models. We show the good ability of the proposed bound to predict the well known threshold effect. We also show the influence of the polarization parameters.
\end{abstract}

Index Terms - DOA and polarization estimation, WeissWeinstein bound, COLD array

\section{INTRODUCTION}

In array processing, the use of an antenna able to handle the source polarization is of importance for a large class of application (see [1] and references therein). One of such antenna is the so-called COLD (cocentered orthogonal loop and dipole) array. Several papers related to the COLD array can be cited: in [1], the authors have introduced an efficient Direction-Of-Arrival (DOA) and polarization estimation technique and the COLD array was shown to improve both the DOA and the polarization estimation performance compared to the cocentered crossed-dipole array. Another example concerning asymptotic performance is given in [2] where the statistical resolution limit, under the conditional observation model (i.e. when the source signals are assumed to be deterministic) was investigated. The authors have introduced an approach based on the Cramér-Rao bound (CRB), and again, under some conditions, the COLD array was shown to provide better performance than a conventional array.

In this paper, we are interested by the ultimate performance of such an array in non-asymptotic scenarios, i.e., when the Signal to Noise Ratio (SNR) and/or the number of snapshots are low. Indeed, it is well known that such an array processing problem is a nonlinear estimation problem for which a threshold phenomenon (i.e., when a drastic increase of the DOA estimator mean square error appears) [3]. Unfortunately, the CRB can not capture this threshold effect [4]. Therefore, we are herein interested to investigate a more relevant bound for the COLD array observation model: the so-called Weiss-Weinstein bound (WWB) [5]. Moreover, since the WWB is a Bayesian bound, it takes into account the support of the parameter via the prior distribution of the parameters. Consequently, the WWB provides a powerful tool to predict the global MSE behavior in both asymptotic and non-asymptotic regions.

This project is funded by both the Digiteo Research Park and the Region Ile-de-France.
Due to its complexity (in comparison with the CRB), there are few publications related to the WWB in the literature. Some of previous works, such as [6], have evaluated the WWB only by way of simulations (i.e., without closed-form expressions). The bound has been compared to the MSE of the MUSIC algorithm and classical Beamforming using an $8 \times 8$ element array antenna. In [7], the authors have introduced a numerical comparison between the Bayesian CRB, the Ziv-Zakai bound and the WWB for DOA estimation. In [8], numerical simulations of the WWB to optimize sensor positions for non-uniform linear arrays have been presented. In [9], by considering the matched-field estimation problem, the authors have derived a semi closed-form expression of the WWB in the socalled unconditional observation model (i.e. when the source signals are assumed to be Gaussian). Concerning the aforementioned context of the conditional observation model a closed-form expression of the WWB is given in the simple case of spectral analysis in [10]. Recently, the closed-form expressions of the WWB for DOA estimation with a classical planar array were derived in [11], and were applied for the array geometry design. Note that all these works have been performed in the context where arrays are not able to handle the polarization of the source signals.

In this paper, we consider the WWB performance bound on the DOA and the SNR threshold analysis of a polarized source with a COLD array. The closed-form expressions of the WWB are given under both conditional and unconditional observation models for which we present simulation results.

\section{MODEL SETUP}

We consider the context of DOA estimation of a single polarized source using a linear (possibly non-uniform) array of $N$ COLD sensors. The source signal is assumed to be narrow-band and to be located in the far-field area. The sensor positions with respect to a reference axis are characterized by the vector $\mathbf{d}=\left[d_{1} \ldots d_{N}\right] . \quad$ As in $[1,12]$ we assume that the source is coplanar with the array. Therefore, the DOA of the source signal depends only on the azimuth angle, denoted $\phi$. We assume that the source polarization is known or previously estimated. Let us set $\mathbf{u}=\left[\frac{j 2 \pi A_{s l}}{\lambda} \cos \rho-L_{s d} \sin \rho e^{(j \psi)}\right]^{T}$, the polarization vector, with polarization angles $\rho \in[0, \pi / 2]$ and $\psi \in[-\pi, \pi]$. $\lambda$ denotes the wavelength, and $A_{s l}, L_{s d}$ denote the dipole lengths and the loop perimeters, respectively, with the assumption that $A_{s l}<3 \lambda / 10$, and $L_{s d}<\lambda / 10$. The output at the $i^{t h}$ sensor and for the $t^{t h}$ snapshot is a two components vector given by [4]:

$$
\left[\begin{array}{c}
\hat{y}_{i}(t) \\
\breve{y}_{i}(t)
\end{array}\right]=[\mathbf{a}(\phi)]_{i} s(t) \mathbf{u}+\mathbf{n}_{i}(t), \quad t=1, \ldots, T
$$


where $s(t)$ denotes the source signal, where $[\mathbf{a}(\phi)]_{i}=e^{\left(j \frac{2 \pi}{\lambda} d_{i} \sin \phi\right)}$ denotes the $i^{t h}$ element of the steering vector $\mathbf{a}(\phi)$ and where $\mathbf{n}_{i}(t)$ is an additive noise. This noise is assumed to be complex, circular, uncorrelated (spatially, temporally and between the loop and the dipole of each sensors) Gaussian with zero mean and the variance $\sigma^{2}$. We will use the two classical alternative hypothesis about the source signal

- $\mathcal{H}_{1}$ : the source signal is assumed to be deterministic and known. This is the so-called conditional signal model [13].

- $\mathcal{H}_{2}$ : the source signal is assumed to be a complex circular random Gaussian vector, independent of the noise, with zero mean and known covariance matrix $\sigma_{s}^{2} \mathbf{I}$. This is the so-called unconditional signal model [13].

For mathematical convenience, we consider the estimation of $\omega=\sin \phi$, and we assume that $\omega$ follows a uniform distribution $\omega \sim \mathcal{U}[-1,1]$, i.e.

$$
p(\omega)=\left[\begin{array}{l}
\frac{1}{2} \text { if }-1 \leq \omega \leq 1, \\
0 \text { otherwise }
\end{array}\right.
$$

The output signal for the $t^{t h}$ snapshot of the whole array can be then expressed as:

$$
\mathbf{y}(t)=\left[\begin{array}{c}
\hat{\mathbf{y}}(t) \\
\breve{\mathbf{y}}(t)
\end{array}\right]=\mathbf{b}(\omega) s(t)+\mathbf{n}(t)
$$

where $\mathbf{b}(\omega)=\mathbf{u} \otimes \mathbf{a}(\omega)$, with $\otimes$ denotes the Kronecker product, where $\hat{\mathbf{y}}(t)=\left[\hat{y}_{1}(t) \ldots \hat{y}_{N}(t)\right]^{T}$, and where $\breve{\mathbf{y}}(t)=\left[\breve{y}_{1}(t) \ldots \breve{y}_{N}(t)\right]^{T}$.

With the aforementioned assumptions, under $\mathcal{H}_{1}$, the full observation vector $(\forall t)$ follows a Gaussian distribution with parameterized mean, i.e., $\mathbf{y} \mid \omega \sim \mathcal{C N}\left(\mathbf{I}_{T} \otimes \mathbf{b}(\omega) \mathbf{s}, \sigma^{2} \mathbf{I}_{2 N T}\right)$, with $\mathbf{s}=$ $[s(1) \ldots s(T)]^{T}$. And, under $\mathcal{H}_{2}, \mathbf{y}(t)$ follows a Gaussian distribution with parameterized covariance matrix, i.e., $\mathbf{y} \mid \omega \sim \mathcal{C N}\left(\mathbf{0}, \mathbf{I}_{T} \otimes\right.$ $\mathbf{R}(\omega)$ ), where $\mathbf{R}(\omega)=\sigma_{s}^{2} \mathbf{b}(\omega) \mathbf{b}(\omega)^{H}+\sigma^{2} \mathbf{I}_{2 N}$ Consequently, the likelihood function under $\mathcal{H}_{1}$ can be expressed as:

$$
p(\mathbf{y} \mid \omega)=\frac{1}{\left(\pi \sigma^{2}\right)^{2 N T}} \exp \left(-\frac{1}{\sigma^{2}} \sum_{t=1}^{T}\|\mathbf{y}(t)-\mathbf{b}(\omega) s(t)\|^{2}\right),
$$

and the likelihood function under $\mathcal{H}_{2}$ is given by

$$
p(\mathbf{y} \mid \omega)=\frac{1}{\pi^{2 N T}|\mathbf{R}(\omega)|^{T}} \exp \left(-\sum_{t=1}^{T} \mathbf{y}(t)^{H} \mathbf{R}(\omega)^{-1} \mathbf{y}(t)\right),
$$

where |.| denotes the matrix determinant and $\|.\|^{2}$ denotes the norm operator. The Weiss-Weinstein bound will be derived under both models $\mathcal{H}_{1}$ and $\mathcal{H}_{2}$.

\section{WEISS-WEINSTEIN BOUNDS FOR THE COLD ARRAY}

The Weiss-Weinstein bound is a lower bound on the mean square error well known to accurately predict the SNR threshold effect which appears in non-linear estimation problems [5]. The WWB is generally obtained by taking the supremum of a function over a set of test point and over a set of parameter $s \in[0,1]$. Concerning the parameter $s$, one often set $s=1 / 2$, see [9-11], although there are no proof that $s=1 / 2$ could leads to the tightest WWB. The WWB for $s=1 / 2$ is given by:

$$
W W B=\sup _{h} \frac{h^{2} \eta(h, 0) \eta(0, h)}{2(\eta(h, h)-\eta(h,-h))}
$$

where $h$ is the difference between the parameter of interest and a test-point and where the function $\eta$ is defined as

$$
\begin{aligned}
\eta(\alpha, \beta) & =\iint_{\Theta} \sqrt{p(\mathbf{y}, \omega+\alpha) p(\mathbf{y}, \omega+\beta)} d \mathbf{y} d \omega \\
& =\int_{\Theta} \sqrt{p(\omega+\alpha) p(\omega+\beta)} \zeta(\alpha, \beta) d \omega,
\end{aligned}
$$

by denoting $\zeta(\alpha, \beta)=\int_{\Omega} \sqrt{p(\mathbf{y} \mid \omega+\alpha) p(\mathbf{y} \mid \omega+\beta)} d \mathbf{y} . \quad \Omega$ and $\Theta$ are the observation space and the parameter space, respectively. $p(\mathbf{y},$.$) and p($.$) denote the joint distribution of the full observation$ vector and the parameter (possibly a test point) and the a priori distribution of the parameter (possibly a test point). Note that one has to respect $\omega+h \in \Theta$.

\subsection{Conditional observation model}

From (4), the expression of $\zeta(\alpha, \beta)$ is given by:

$$
\begin{aligned}
& \zeta(\alpha, \beta)=\int_{\Omega} \frac{1}{\left(\pi \sigma^{2}\right)^{2 N T}} \\
& \times e^{\left(-\frac{1}{2 \sigma^{2}} \sum_{t=1}^{T}\left(\|\mathbf{y}(t)-\mathbf{b}(\omega+\alpha) s(t)\|^{2}+\|\mathbf{y}(t)-\mathbf{b}(\omega+\beta) s(t)\|^{2}\right)\right)} d \mathbf{y} .
\end{aligned}
$$

By substituting $\mathbf{x}(t)=\mathbf{y}(t)-\frac{1}{2}(\mathbf{b}(\omega+\alpha) s(t)+\mathbf{b}(\omega+\beta) s(t))$, it easily leads to

$$
\begin{aligned}
& -\frac{1}{2 \sigma^{2}} \sum_{t=1}^{T}\left(\|\mathbf{y}(t)-\mathbf{b}(\omega+\alpha) s(t)\|^{2}+\|\mathbf{y}(t)-\mathbf{b}(\omega+\beta) s(t)\|^{2}\right) \\
& =-\frac{1}{\sigma^{2}} \sum_{t=1}^{T}\left(\|\mathbf{x}\|^{2}+\frac{1}{4}\|\mathbf{b}(\omega+\alpha)-\mathbf{b}(\omega+\beta)\|^{2}\right)
\end{aligned}
$$

Since

$$
\int_{\Omega} \frac{1}{\left(\pi \sigma^{2}\right)^{2 N T}} \exp \left(-\frac{1}{\sigma^{2}}\|\mathbf{x}\|^{2}\right) d \mathbf{x}=1
$$

one obtains

$$
\zeta(\alpha, \beta)=\exp \left(-\frac{\|\mathbf{s}\|^{2}}{4 \sigma^{2}}\|\mathbf{b}(\omega+\alpha)-\mathbf{b}(\omega+\beta)\|^{2}\right) .
$$

Since $\mathbf{b}(\omega)=\mathbf{u} \otimes \mathbf{a}(\omega)$, and since $\mathbf{u}^{H} \mathbf{u}=\frac{4 \pi^{2} A_{s l}^{2}}{\lambda^{2}} \cos ^{2} \rho+$ $L_{s d}^{2} \sin ^{2} \rho$, the closed-form expression of $\|\mathbf{b}(\omega+\alpha)-\mathbf{b}(\omega+\beta)\|^{2}$ is obtained by noting that

$$
\begin{aligned}
\|\mathbf{b}(\omega+\alpha)\|^{2}= & \|\mathbf{b}(\omega+\beta)\|^{2} \\
= & N\left(\frac{4 \pi^{2} A_{s l}^{2}}{\lambda^{2}} \cos ^{2} \rho+L_{s d}^{2} \sin ^{2} \rho\right), \\
\mathbf{b}(\omega+\alpha)^{H} \mathbf{b}(\omega+\beta)= & \left(\frac{4 \pi^{2} A_{s l}^{2}}{\lambda^{2}} \cos ^{2} \rho+L_{s d}^{2} \sin ^{2} \rho\right) \\
& \times \sum_{i=1}^{N} e^{\left(j \frac{2 \pi}{\lambda} d_{k}(\beta-\alpha)\right)},
\end{aligned}
$$

and that

$$
\begin{aligned}
\mathbf{b}(\omega+\beta)^{H} \mathbf{b}(\omega+\alpha)= & \left(\frac{4 \pi^{2} A_{s l}^{2}}{\lambda^{2}} \cos ^{2} \rho+L_{s d}^{2} \sin ^{2} \rho\right) \\
& \times \sum_{i=1}^{N} e^{\left(j \frac{2 \pi}{\lambda} d_{k}(\alpha-\beta)\right)} .
\end{aligned}
$$


One observes that the functions $\zeta(\alpha, \beta)$ are no longer depending on the parameter $\omega$, consequently, $\eta(\alpha, \beta)$ can be rewritten as:

$$
\eta(\alpha, \beta)=\zeta(\alpha, \beta) \int_{\Theta} \sqrt{p(\omega+\alpha) p(\omega+\beta)} d \omega .
$$

Under the uniform distribution prior assumption, one obtains:

$$
\int_{\Theta} \sqrt{p(\omega+\alpha) p(\omega+\beta)}=1-\frac{|\alpha|+|\beta|}{2} .
$$

From (6), (12), (13), (14), (15) and (16), one obtains the closedexpression of WWB as (17) (shown on the top of next page). Note that the bound is independent of the parameter $\psi$.

\subsection{Unconditional observation model}

From (5), the expression of $\zeta(\alpha, \beta)$ can be expressed as:

$$
\begin{aligned}
\zeta(\alpha, \beta)= & \int_{\Omega} \frac{1}{\pi^{2 N T}|\mathbf{R}(\omega+\alpha)|^{T / 2}|\mathbf{R}(\omega+\beta)|^{T / 2}} \\
& \times e^{\left(-\sum_{t=1}^{T} \mathbf{y}(t)^{H}\left(\frac{\mathbf{R}(\omega+\alpha)^{-1}+\mathbf{R}(\omega+\beta)-1}{2}\right) \mathbf{y}(t)\right)} d \mathbf{y}
\end{aligned}
$$

By setting $\boldsymbol{\Gamma}^{-1}=\frac{\mathbf{R}(\omega+\alpha)^{-1}+\mathbf{R}(\omega+\beta)^{-1}}{2}$, one obtains, $|\boldsymbol{\Gamma}|=$ $\frac{2^{2 N}}{\left|\mathbf{R}(\omega+\alpha)^{-1}+\mathbf{R}(\omega+\beta)^{-1}\right|}$, which leads to

$$
\begin{aligned}
\zeta(\alpha, \beta)= & \frac{|\boldsymbol{\Gamma}|^{T}}{|\mathbf{R}(\omega+\alpha)|^{T / 2}|\mathbf{R}(\omega+\beta)|^{T / 2}} \\
& \times \int_{\Omega} \frac{1}{\pi^{2 N T}|\boldsymbol{\Gamma}|^{T}} \exp \left(-\sum_{t=1}^{T} \mathbf{y}(t)^{H} \boldsymbol{\Gamma}^{-1} \mathbf{y}(t)\right) d \mathbf{y} .
\end{aligned}
$$

Since $\int_{\Omega} \frac{1}{\pi^{2 N T}|\boldsymbol{\Gamma}|^{T}} \exp \left(-\sum_{t=1}^{T} \mathbf{y}(t)^{H} \boldsymbol{\Gamma}^{-1} \mathbf{y}(t)\right) d \mathbf{y}=1$, one obtains

$$
\zeta(\alpha, \beta)=\frac{|\boldsymbol{\Gamma}|^{T}}{|\mathbf{R}(\omega+\alpha)|^{T / 2}|\mathbf{R}(\omega+\beta)|^{T / 2}} .
$$

Due to the special structure of $\mathbf{R}(\omega+\delta)=\sigma_{s}^{2} \mathbf{b}(\omega+\delta) \mathbf{b}(\omega+\delta)^{H}+$ $\sigma^{2} \mathbf{I}_{2 N}$, one easily gets

$$
|\mathbf{R}(\omega+\delta)|=\sigma^{4 N}\left(1+\frac{\sigma_{s}^{2}}{\sigma^{2}}\|\mathbf{b}(\omega+\delta)\|^{2}\right) .
$$

Furthermore, thanks to Woodbury identity, one gets

$$
\mathbf{R}(\omega+\delta)^{-1}=\frac{1}{\sigma^{2}}\left(\mathbf{I}_{2 N}-\frac{\sigma_{s}^{2} \mathbf{b}(\omega+\delta) \mathbf{b}(\omega+\delta)^{H}}{\sigma_{s}^{2}\|\mathbf{b}(\omega+\delta)\|^{2}+\sigma^{2}}\right),
$$

thus

$$
\begin{aligned}
& \mathbf{R}(\omega+\alpha)^{-1}+\mathbf{R}(\omega+\beta)^{-1}= \\
& \frac{1}{\sigma^{2}}\left(2 \mathbf{I}_{2 N}-\frac{\sigma_{s}^{2} \mathbf{b}(\omega+\alpha) \mathbf{b}(\omega+\alpha)^{H}}{\sigma_{s}^{2}\|\mathbf{b}(\omega+\alpha)\|^{2}+\sigma^{2}}-\frac{\sigma_{s}^{2} \mathbf{b}(\omega+\beta) \mathbf{b}(\omega+\beta)^{H}}{\sigma_{s}^{2}\|\mathbf{b}(\omega+\beta)\|^{2}+\sigma^{2}}\right) .
\end{aligned}
$$

The matrix determinant of $\mathbf{R}(\omega+\alpha)^{-1}+\mathbf{R}(\omega+\beta)^{-1}$ equals to the product of their eigenvalues. Particularly, there are $2 N-2$ eigenvalues equal to $2 / \sigma^{2}$, and the eigenvectors corresponding to the two remaining eigenvalues is a linear combination of the form $\mathbf{b}(\omega+\alpha)+q \mathbf{b}(\omega+\beta)$. Furthermore, the two above eigenvalues $\nu$ are the solution of the following equation

$$
\begin{aligned}
& \left(\mathbf{R}(\omega+\alpha)^{-1}+\mathbf{R}(\omega+\beta)^{-1}\right)(\mathbf{b}(\omega+\alpha)+q \mathbf{b}(\omega+\beta)) \\
& =\nu(\mathbf{b}(\omega+\alpha)+q \mathbf{b}(\omega+\beta)),
\end{aligned}
$$

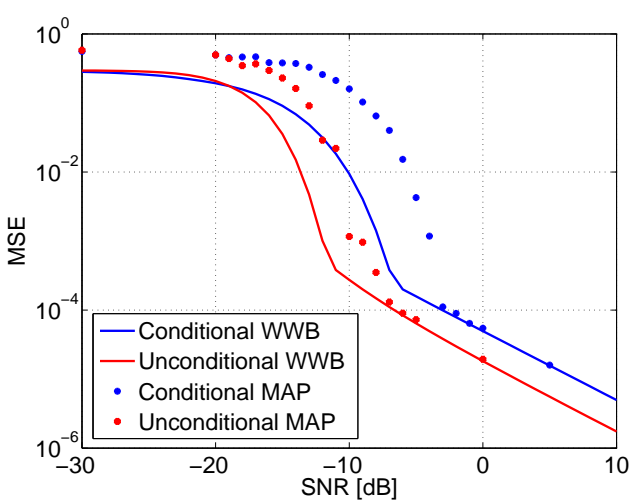

Fig. 1. MAP versus WWB.

which reduces to

$$
\begin{aligned}
& \mathbf{b}(\omega+\alpha)\left(\frac{1}{\sigma^{2}}\left(2-A\|\mathbf{b}(\omega+\alpha)\|^{2}-q A C\right)-\nu\right) \\
& +\mathbf{b}(\omega+\beta)\left(\frac{1}{\sigma^{2}}\left(2 q-B q\|\mathbf{b}(\omega+\beta)\|^{2}-B C^{H}\right)-q \nu\right)=0
\end{aligned}
$$

where $A=\frac{\sigma_{s}^{2}}{\sigma_{s}^{2}\|\mathbf{b}(\omega+\alpha)\|^{2}+\sigma^{2}}, B=\frac{\sigma_{s}^{2}}{\sigma_{s}^{2}\|\mathbf{b}(\omega+\beta)\|^{2}+\sigma^{2}}$ and $C=$ $\mathbf{b}(\omega+\alpha){ }^{H} \mathbf{b}(\omega+\beta)$. Since $\mathbf{b}(\omega+\alpha)$ and $\mathbf{b}(\omega+\beta)$ are nonlinear, thus, their coefficients are equal to 0 . Solving the first coefficient for $q$ and then substituting $q$ into the second coefficient one obtains the equation

$$
\begin{aligned}
& \nu^{2} \sigma^{4}+\nu \sigma^{2}\left(2-A\|\mathbf{b}(\omega+\alpha)\|^{2}-2+B\|\mathbf{b}(\omega+\beta)\|^{2}\right) \\
& -4+2 A\|\mathbf{b}(\omega+\alpha)\|^{2}+2 B\|\mathbf{b}(\omega+\beta)\|^{2} \\
& -A B\|\mathbf{b}(\omega+\alpha)\|^{2}\|\mathbf{b}(\omega+\beta)\|^{2}+A B C C^{H}=0 .
\end{aligned}
$$

Solving (26) for $\nu$, and since $\|\mathbf{b}(\omega+\alpha)\|^{2}=\|\mathbf{b}(\omega+\beta)\|^{2}=$ $\|\mathbf{b}(\omega)\|^{2}$, one obtains

$$
\begin{aligned}
& \left|\mathbf{R}(\omega+\alpha)^{-1}+\mathbf{R}(\omega+\beta)^{-1}\right|=\prod_{i=1}^{2 N} \nu_{i} \\
& \quad=\frac{2^{2 N}}{\sigma^{4 N}}\left(\frac{\sigma^{2}}{\|\mathbf{b}(\omega)\|^{2} \sigma_{s}^{2}+\sigma^{2}}+\frac{1}{4} \frac{\sigma_{s}^{4}\left(\|\mathbf{b}(\omega)\|^{4}-\|C\|^{2}\right)}{\left(\|\mathbf{b}(\omega)\|^{2} \sigma_{s}^{2}+\sigma^{2}\right)^{2}}\right) .
\end{aligned}
$$

Finally, substituting (21), (27) into (20), we have

$\zeta(\alpha, \beta)=\left(1+\frac{\sigma_{s}^{2}\left(\|\mathbf{b}(\omega)\|^{4}-\left\|\mathbf{b}(\omega+\alpha)^{H} \mathbf{b}(\omega+\beta)\right\|^{2}\right)}{4 \sigma^{2}\left(\|\mathbf{b}(\omega)\|^{2} \sigma_{s}^{2}+\sigma^{2}\right)}\right)^{-T}$.

From (12), (13), and (14), one observes that, as for the conditional case, $\zeta(\alpha, \beta)$ does not depends on the parameter $\omega$. Consequently, the closed-form expression of the WWB is given by (29) (shown on the top of next page). Note that the bound is independent of the parameter $\psi$.

\section{SIMULATION RESULT}

In a first simulation, we compare the MSE behavior of the maximum a posteriori estimator (MAP) versus the WWB. We use an uniform linear COLD array consisting of $N=10$ sensors with the intersensors spacing equal to $\lambda / 2$ and $T=20$ snapshots. The polarization parameter are set to $\rho=\pi / 4, \psi=\pi / 3, A_{s l}=2 \lambda / 10$, and $L_{s d}=\lambda / 10$. The empirical MSE of the MAP is obtained over 1000 


$$
W W B=\sup _{h} \frac{h^{2}\left(1-\frac{|h|}{2}\right)^{2} \exp \left(-\frac{\|\mathbf{s}\|^{2}}{\sigma^{2}}\left(\frac{4 \pi^{2} A_{s l}^{2}}{\lambda^{2}} \cos ^{2} \rho+L_{s d}^{2} \sin ^{2} \rho\right)\left(N-\sum_{k=1}^{N} \cos \left(\frac{2 \pi}{\lambda} d_{k} h\right)\right)\right)}{2\left(1-\frac{|h|}{2}\right)-2(1-|h|) \exp \left(-\frac{\|\mathbf{s}\|^{2}}{2 \sigma^{2}}\left(\frac{4 \pi^{2} A_{s l}^{2}}{\lambda^{2}} \cos ^{2} \rho+L_{s d}^{2} \sin ^{2} \rho\right)\left(N-\sum_{k=1}^{N} \cos \left(\frac{4 \pi}{\lambda} d_{k} h\right)\right)\right)} .
$$

$$
W W B=\sup _{h} \frac{h^{2}\left(1-\frac{|h|}{2}\right)^{2}\left(1+\frac{\sigma_{s}^{2}\left(\frac{4 \pi^{2} A_{s l}^{2}}{\lambda^{2}} \cos ^{2} \rho+L_{s d}^{2} \sin ^{2} \rho\right)^{2}\left(N^{2}-\left\|\sum_{k=1}^{N} \exp \left(j \frac{2 \pi}{\lambda} d_{k} h\right)\right\|^{2}\right)}{4 \sigma^{2}\left(N\left(\frac{4 \pi^{2} A_{s l}^{2}}{\lambda^{2}} \cos ^{2} \rho+L_{s d}^{2} \sin ^{2} \rho\right) \sigma_{s}^{2}+\sigma^{2}\right)}\right)^{-2 T}}{2\left(1-\frac{|h|}{2}\right)-2(1-|h|)\left(1+\frac{\sigma_{s}^{2}\left(\frac{4 \pi^{2} A_{s l}^{2}}{\lambda^{2}} \cos ^{2} \rho+L_{s d}^{2} \sin ^{2} \rho\right)^{2}\left(N^{2}-\left\|\sum_{k=1}^{N} \exp \left(j \frac{4 \pi}{\lambda} d_{k} h\right)\right\|^{2}\right)}{4 \sigma^{2}\left(N\left(\frac{\left.\left.4 \pi^{2} A_{s l}^{2} \cos ^{2} \rho+L_{s d}^{2} \sin ^{2} \rho\right) \sigma_{s}^{2}+\sigma^{2}\right)}{\lambda^{2}}\right)^{-T}\right.} .\right.}
$$
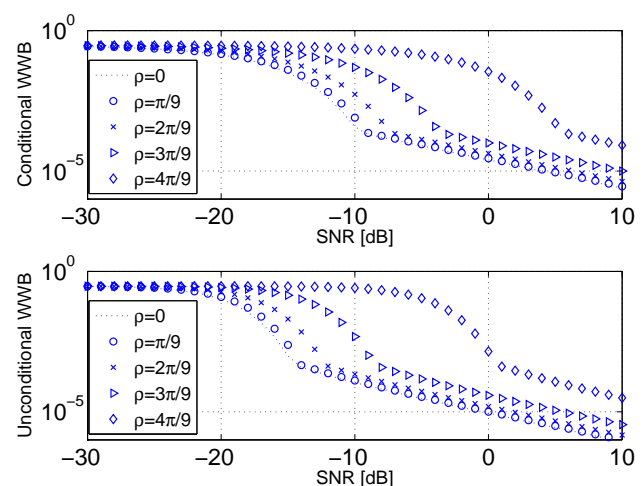

Fig. 2. WWB w.r.t the parameter $\rho$

Monte Carlo trials. Fig. 1 shows that the WWB is a tight bound, which well capture the SNR threshold of the MAP MSE (around 3 $[\mathrm{dB}])$.

On the other hand, the impact of the polarization parameter, $\rho$, is investigated. The scenario is the same as the previous simulation. Fig. 2 shows the WWB versus the SNR, according to different value of $\rho$ under both assumptions. One observes that in each cases, both the SNR threshold and asymptotic MSE are affected by $\rho$.

\section{CONCLUSION}

In this paper, we have derived closed-expressions of the WWB in the context of source localization with a COLD array under both conditional and unconditional observation models. The WWB is shown to be a useful tool to capture the threshold effect. Furthermore, we showed that the polarization parameter $\rho$ has a strongly impact on the MSE behavior.

\section{REFERENCES}

[1] J. Li, P. Stoica, and D. Zheng, "Efficient direction and polarization estimation with a COLD array," IEEE Trans. Antennas Propagat., vol. 44, no. 4, pp. 539-547, Apr. 1996.

[2] M. N. El Korso, R. Boyer, A. Renaux, and S. Marcos, "Statisti- cal resolution limit of the uniform linear cocentered orthogonal loop and dipole array," vol. 59, no. 1, pp. 425-431, 2011.

[3] H. L. VanTrees and K. L. Bell, Bayesian bounds for parameter estimation and nonlinear filtering/tracking. New York: Wiley, 2007.

[4] R. Boyer, "Analysis of the cold uniform linear array," in Proc. IEEE Int. Work. Signal Processing, Wireless Communications, Perugia, Italy, 2009.

[5] E. Weinstein and A. J. Weiss, "A general class of lower bounds in parameter estimation," IEEE Trans. Inform. Theory, vol. 34, pp. 338-342, Mar. 1988.

[6] T. J. Nohara and S. Haykin, "Application of the WeissWeinstein bound to a two dimensional antenna array," in IEEE Trans. Acoust., Speech, Signal Processing, vol. 36, no. 9, Sep. 1988, pp. 1533-1534.

[7] H. Nguyen and H. L. VanTrees, "Comparison of performance bounds for DOA estimation," in IEEE Seventh SP Workshop on Statistical Signal and Array Processing, 1994, pp. 313-316.

[8] F. Athley, "Optimization of element positions for direction finding with sparse arrays," Proceedings of the 11th IEEE Signal Processing Workshop on statistical signal processing, pp. $516-519,2001$.

[9] W. Xu, A. B. Baggeroer, and C. D. Richmond, "Bayesian bounds for matched-field parameter estimation," IEEE Trans. Signal Processing, vol. 52, pp. 3293-3305, Dec. 2004.

[10] A. Renaux, "Weiss-Weinstein bound for data aided carrier estimation," IEEE Signal Processing Lett., vol. 14, no. 4, pp. $283-$ 286, Apr. 2007.

[11] D. T. Vu, A. Renaux, R. Boyer, and S. Marcos, "Closed-form expression of the Weiss-Weinstein bound for 3D source localization: the conditional case," in Proc. IEEE Sensor Array and Multichannel Signal Processing Workshop, Kibutz Ma'ale Hahamisha, Israel, Oct. 2010.

[12] J. Li, "Direction and polarization estimation using arrays with small loops and short depoles," IEEE Trans. Antennas Propagat., vol. 41, no. 3, pp. 379-387, Mar. 1993.

[13] B. Ottersten, M. Viberg, P. Stoica, and A. Nehorai, "Exact and large sample maximum likelihood techniques for parameter estimation and detection in array processing," in Radar Array Processing, S. Haykin, J. Litva, and T. J. Shepherd, Eds. Berlin: Springer-Verlag, 1993, ch. 4, pp. 99-151. 